\title{
Radio detection of extensive air showers
}

\section{Measurements of the properties of cosmic rays with the radio technique - past, present, future}

\author{
Jörg R. Hörandel ${ }^{1,2,3, \star}$ \\ ${ }^{1}$ Radboud Universiteit, Department of Astrophysics/IMAPP, P.O. Box 9010, 6500 GL Nijmegen, The Nether- \\ lands \\ ${ }^{2}$ Nikhef, Science Park 105, 1098 XG Amsterdam, The Netherlands \\ ${ }^{3}$ Vrije Universiteit Brussel, Department of Physics and Astronomy, B-1050 Brussels, Belgium
}

\begin{abstract}
Radio detection of extensive air showers is a flourish technique, attracting more and more interest to investigate the properties of high-energy cosmic rays. Past, present, and future developments are reviewed.
\end{abstract}

\section{Historical remarks}

The idea of radio detection of extensive air showers started in the 1940s at Jodrell Bank [1]. Blackett and Lovell suggested detecting air showers by the means of the radar echoes from the trail of ionization left behind the shower front as it passes though the atmosphere. Experiments in 1946 used surplus military radars. They failed to detect air showers but instead, they found high-altitude trails of meteors. This work led to the development of radio astronomy at Jodrell Bank [2].

John Jelley suggested in 1958 that air showers itself may emit radiation at radio wavelengths [3]. In the 1960s Porter and Jelley built a small particle detector array and antennas at "Blackett's field" at Jodrell Bank. The particle detectors were three trays of Geiger counters located on a $50 \mathrm{~m}$ triangle build by Trevor Weeks, later a pioneer in gamma-ray astronomy. The radio antenna was a $6 \lambda \times 6 \lambda$ array of dipoles operating at $44 \mathrm{MHz}$. This frequency was used throughout the day by BBC TV and was quiet at night time. First radio signals from air showers were recorded by Jelley and Porter around $1965[4,5]$. Coincident pulses from the counters triggered an oscilloscope fitted with a recording camera. The radio signals from the antenna were delayed to allow for delays in the triggering system, filtered, amplified and the power measured before being displayed on the oscilloscope.

Harold Allan's group set up radio antennas, operating at frequencies of 32, 44, and $60 \mathrm{MHz}$ at the Haverah Park air shower experiment from the University of Leeds in the late 1960s, see Fig. (1) (left). The right-hand panel of Fig. (1) shows Harold Allan lecturing at the 1st European Symposium on High Energy Interactions and Extensive Air Showers, Lodz, Poland in April 1968. The early work on the radio detection of air showers is summarized by Allan in an excellent review [6]. The review presented the lateral distribution of the electric field from the radio emission of air showers

$$
\epsilon_{v}=2\left(\frac{E_{p}}{10^{17} \mathrm{eV}}\right)\left(\frac{\sin \alpha \cos \theta}{\sin 45^{\circ} \cos 30^{\circ}}\right) \exp \left(\frac{-r}{r_{0}}\right)\left(\frac{v}{50 \mathrm{MHz}}\right)^{-1} \mu \mathrm{V} / \mathrm{m} / \mathrm{MHz}
$$

\footnotetext{
^e-mail: j.horandel@astro.ru.nl
} 

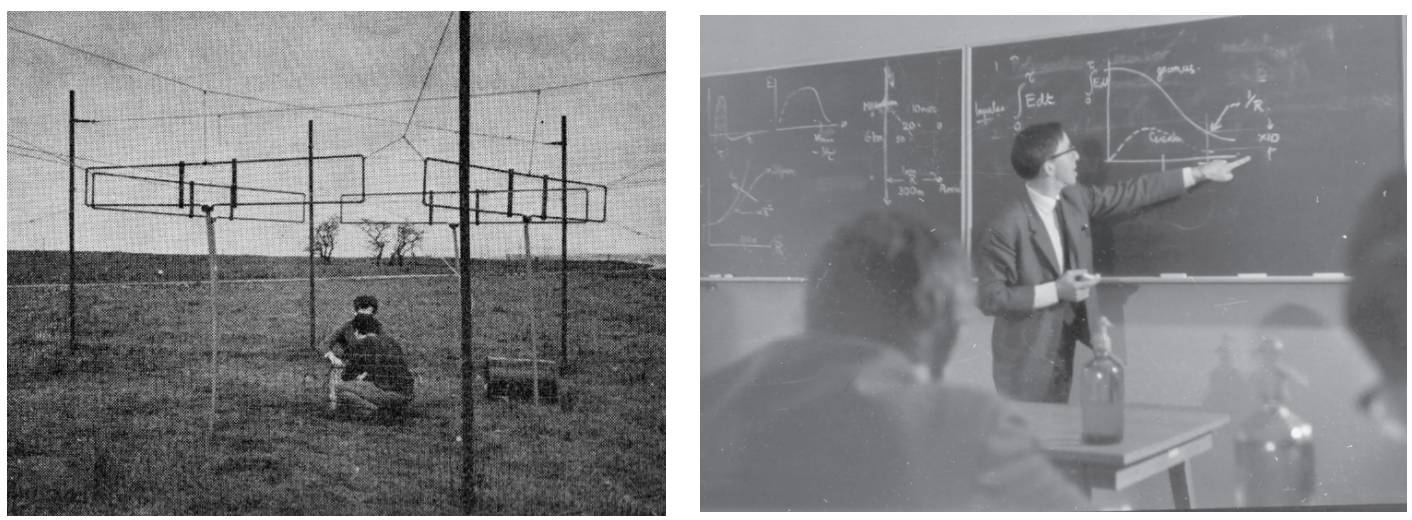

Figure 1. Left: Roger Clay and Keith Jones working at the radio antennas at the Haverah Park extensive air shower array. Right: Harold Allan lecturing at the 1st European Symposium on High Energy Interactions and Extensive Air Showers, Lodz, Poland, April 1968 (courtesy Alan Watson).

where $R_{0}=110 \mathrm{~m}$ at $v=55 \mathrm{MHz}$. This lateral distribution was used for decades until recently precision measurements and simulations allowed the determination of more detailed numerical descriptions $[7,8]$. The interest in the radio detection of air showers declined in the 1970s. It experienced a renaissance after the year 2000 [9] when "modern" experiments such as CODALEMA [10] and LOPES [11] entered the scene.

\section{Actual status and recent results}

In recent years the interest in the radio detection of air showers is strongly growing $[12,13]$. Activities around the world include the LOFAR key science project Cosmic Rays [14, 15]; the Auger Engineering Radio Array (AERA) at the Auger Observatory [16]; in Siberia installations at Yakutsk [17] and the Tunka valley [18]; and ARIANNA [19] on the Ross Ice Shelf in Antarctica. Significant efforts are made to calibrate the response of those detection systems with high precision, both for the signal strength (e.g. [20, 21]) and the signal arrival time (e.g. [22, 23]).

\subsection{Emission processes}

LOFAR has a very dense antenna spacing in its core [25], which is very useful to examine details of the radio emission processes in the atmosphere. There is now consensus that the bulk of the radiation originates from the transverse separation of charges in the shower due to interactions with the Earth magnetic field: the geomagnetic effect [26]. One expects the radiation intensity to be proportional to $v \times B$, where $v$ denotes the direction of the particles in the shower (shower axis) and $B$ the Earth magnetic field. This yields to a north-south asymmetry in the arrival direction of air showers with a strong radiation signal, as e.g. observed by LOFAR in the frequency band $30-80$ [20] and $110-$ $190 \mathrm{MHz}$ [14]. CODALEMA has measured a clear correlation between the effective field strength and the relation $v \times B$ [24], see Fig. (2) (left). The geomagnetic radiation is linearly polarized.

A second, sub-dominant component arises through the longitudinal separation of charges in the shower (Askaryan effect [27]). This component is radially polarized. The direction of the electric field within the shower is measured with high resolution with LOFAR [28]. This allows one to quantitatively evaluate the contribution of the two emission processes to the overall shower emission. The results yield a dependence on the zenith angle and the distance to the shower axis for the ratio of the 

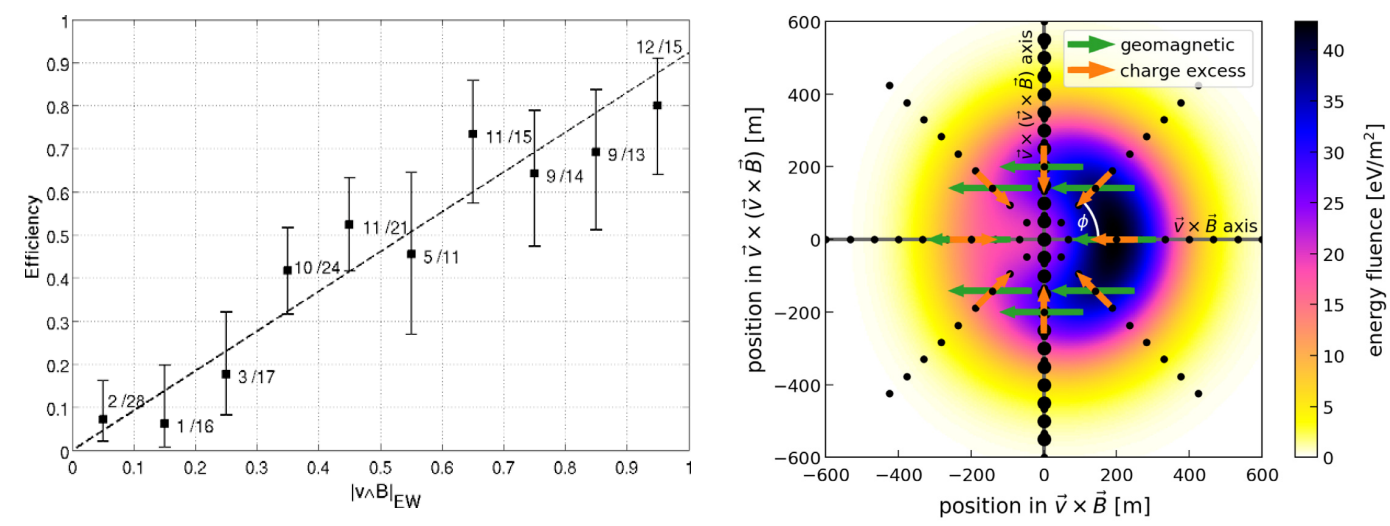

Figure 2. Left: Number of measured air showers recorded with radio emission relative to the number of showers recorded by scintillators with energies exceeding $10^{17} \mathrm{eV}$ as a function of $v \times B$ [24]. Right: Footprint of the energy fluence of the radio emission from an air shower. The arrows indicate the polarization direction of the two emission components [8].

two radiation components. Values for the ratio range from about $5 \%$ to $20 \%$, with an average contribution of the Askaryan effect of about $15 \%$. A similar value has been obtained through polarization measurements with AERA [29].

Interference between the two radiation components with their different polarization behavior leads to a non-rotational symmetric distribution of the radiation intensity on the ground, as illustrated in Fig. (2) (right) and e.g. observed by CODALEMA [30]. This directly implies a non-trivial behavior of the lateral density distribution of the radio emission. Measurements with the relatively small and sparse LOPES array were puzzling [31]; lateral distributions with "unusual shapes" have been found. The break through came with measurements, using the dense LOFAR core [7, 32]. It became obvious that the lateral density distribution is not rotationally symmetric and a simple description as e.g. equation (1) is not sufficient. Instead now, more complex, two-dimensional functions are being used to describe the azimuthal dependence of the radiation strength $[7,8]$.

The detailed measurements also stimulated progress in the theoretical understanding of the radio emission from air showers. Standard simulation tools are now at hand to calculate the radio emission induced by cosmic rays such as the CORSIKA/CoREAS code [33, 34] or ZHAireS [35].

\subsection{Properties of cosmic rays}

The ultimate goal of the radio measurements of air showers is to derive the properties of the incoming cosmic rays, namely their arrival direction, energy and particle type (nuclear mass).

The direction of the cosmic ray is obtained through a precise measurement of the arrival time of the wavefront at the individual antennas of an array. Again LOFAR, with its high antenna density was valuable to clearly understand the precise shape of the radio wavefront in air showers [36] and it could be shown that a hyperboloid is an appropriate analytical description. This confirms earlier work by LOPES [37]. From measurements of the arrival time of the shower front the direction of the cosmic rays can be determined with a resolution of better than $1^{\circ}$.

The energy of the air shower is derived from the measurement of the radio energy density on the ground. The integral of the lateral density distribution yields the total energy delivered by the air shower to the ground in the frequency band of interest. This quantity is proportional to the shower 

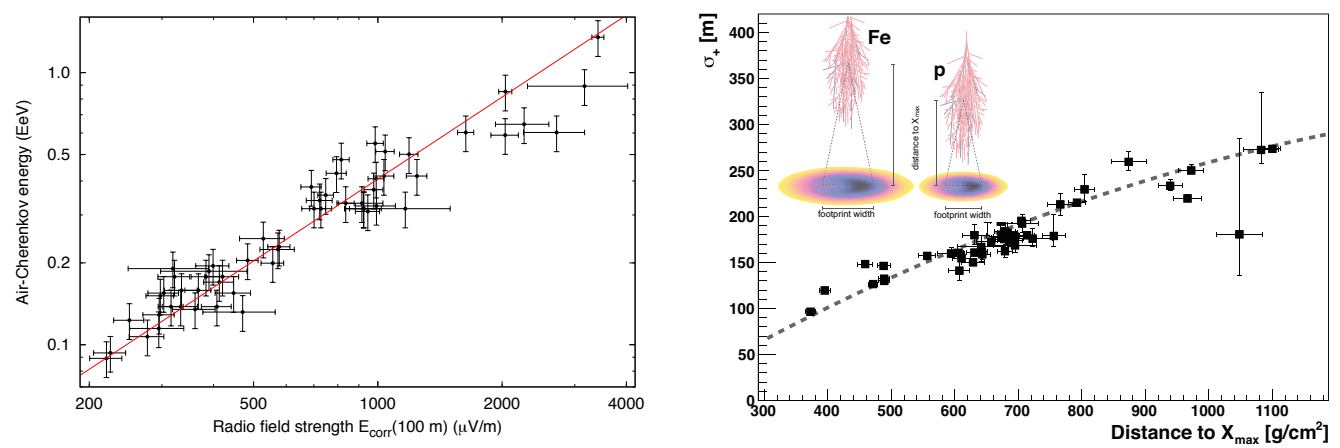

Figure 3. Left: Correlation of the energy measured with the non-imaging air Čerenkov array and the radio antennas at the Tunka site [18]. Right: size of the air shower footprint as a function of the distance from the antennas to the shower maximum [32]. The inset illustrates the underlying principle for air showers induced by a proton and an iron nucleus.

energy. At LOFAR, using results from simulations and a comparison to measurements from a particle detector array [38, 39] an energy resolution around 30\% is obtained [32, 40]. At the Auger observatory the shower energies obtained by the radio measurements have been compared to the energies measured with the well established Auger Surface Detector array. For a set of high-quality showers an energy resolution of better than $25 \%$ has been achieved [41, 42]. At the Tunka experiment, a good correlation is found between the shower energy measured with the non-imaging air Čerenkov detectors and the radio antennas [18], as illustrated in Fig. (3) (left).

Experimentally, the most challenging subject is the determination of the particle type or its nuclear mass. The basic observable in air shower physics is the depth of the shower maximum $X_{\max }$ being proportional to the nuclear mass $A$ of the cosmic ray $X_{\max } \propto \ln A$. The radio signals are in principle emitted on the Čerenkov cone. Thus, the size of the footprint of the radio emission on the ground scales well with the distance from the emission region (basically $X_{\max }$ ) to the antennas (on the ground) [32]. This is depicted in Fig. (3) (right). Knowing the vertical profile of the atmosphere, the depth of the shower maximum $X_{\max }$ can be derived. For LOFAR a high-precision method has been developed to measure $X_{\max }$ [43][15]. For each measured cosmic ray, dedicated simulations are conducted, taking into account the measured energy and direction of the cosmic ray. The predicted signals in the particle and radio detectors are compared to the measured values on a statistical basis and a best fit value is obtained for $X_{\max }$. A resolution for $X_{\max }$ of better than $20 \mathrm{~g} / \mathrm{cm}^{2}$ has been obtained.

This method is also applied to AERA data. The obtained $X_{\max }$ values from the radio measurements have been compared to $X_{\max }$ obseravtions from the fluorescence light telescopes. In first analyses a resolution of the order of $40 \mathrm{~g} / \mathrm{cm}^{2}$ has been achieved. The resolution depends on the number of antennas participating in a measurement and thus, on the quality selection cuts applied [44].

At Tunka a different method is used [45]: The slope of the radio lateral distribution at a distance of $180 \mathrm{~m}$ from the shower axis is found to be sensitive to $X_{\max }$. The obtained values have been correlated with the measurements from the non-imaging Čerenkov detectors and a resolution of the order of $40 \mathrm{~g} / \mathrm{cm}^{2}$ has been obtained.

The described geometrical approach to derive $X_{\max }$ works well for showers with small zenith angles. For showers with large zenith angles, the distance from $X_{\max }$ to the observer is quite large and the expected angular differences are below the experimental resolution. Thus, to measure the mass composition of horizontal air showers a different approach is used [46]: the electron-to-muon ratio is measured with a combination of radio and muon detectors. 


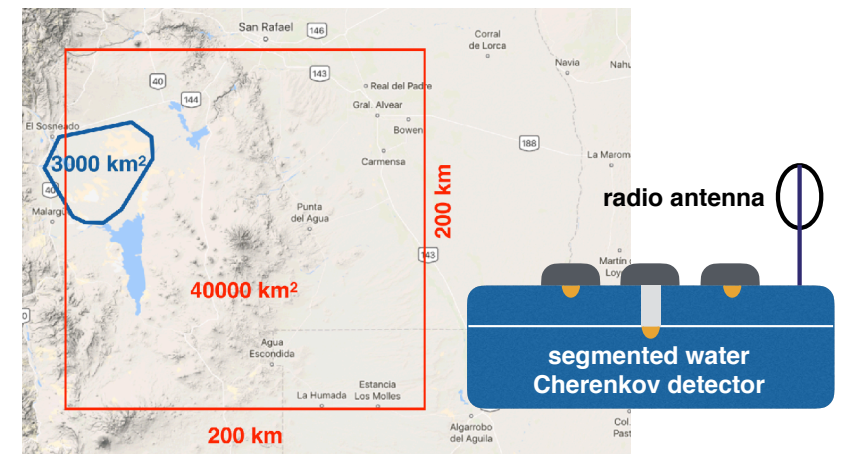

Figure 4. Dream of the author about a next-generation cosmic-ray experiment, covering an area of $40000 \mathrm{~km}^{2}$ with an array of segmented water-Čerenkov detectors and radio antennas.

Combining the various efforts around the world one can state that the radio detection technique is now mature and the properties of cosmic rays are now being measured on a regular basis with such devices with state-of-the-art accuracies of the order of $0.1^{\circ}$ to $0.5^{\circ}$ for the arrival direction, $20 \%$ to $30 \%$ for the energy, and 20 to $40 \mathrm{~g} / \mathrm{cm}^{2}$ for the depth of the shower maximum (particle type), with the accuracies mainly depending on the antenna density of the respective installation.

\section{Future developments}

Radio detection of air showers has a prosperous future ahead. Various activities are planed around the world. Among them are the following projects:

The Square Kilometer Array (SKA) will provide ten thousands of antennas, operating in the frequency band $50-350 \mathrm{MHz}$ on an area of about $1 \mathrm{~km}^{2}[47,48]$. The extreme high antenna density will allow precision measurements of the details of the radio emission from showers and the properties of cosmic rays, in particular a precise determination of $X_{\max }$, a resolution of the order of $10 \mathrm{~g} / \mathrm{cm}^{2}$ has been estimated.

There are plans to enhance the IceCube surface array by scintillation and radio detectors. Simulations predict that by using a broader frequency band the threshold can be lowered to the PeV range $[49,50]$.

The ARIANNA experiment at the Ross Ice Shelf in Antarctica investigates radio emission in a broad frequency range from 50 to $1000 \mathrm{MHz}$ [19]. The broad frequency range is very valuable to derive the properties of air showers recorded by single stations.

GRAND is an ambitious idea to measure highest-energy neutrinos and cosmic rays with a gigantic array, covering $200000 \mathrm{~km}^{2}$ [51]. Neutrinos are expected to interact in mountains and initiate horizontal air showers, which are detected through their radio emission.

At present, the biggest radio detector for air showers is under construction at the Pierre Auger observatory. All Surface Detector stations of the observatory will be equipped with radio antennas, forming a $3000 \mathrm{~km}^{2}$ array to measure the properties of the highest energy cosmic rays [46, 52].

My personal dream for the next step in the investigation of the highest-energy particles in nature is a next-generation cosmic-ray experiment, around the existing Auger observatory as illustrated in Fig. (4). Such an idea would make sense if the upgraded Auger observatory [53] finds a fraction of more than $10 \%$ protons in cosmic rays at the highest energies. The next-generation experiment should have an area at least ten times the surface of the Auger observatory. It could comprise about 10000 detector stations with a spacing of $2 \mathrm{~km}$ on an area of $200 \times 200 \mathrm{~km}^{2}$. The stations could consist of segmented water-Čerenkov detectors (as developed for the Auger project by Letessier-Selvon et al. [54]) and radio antennas on top (as for the upgrade of the Auger observatory). Good mass sensitivity 
will be critical. This will be achieved through electron-muon measurements for vertical showers with the segmented water-Čerenkov detector and for horizontal showers with a combination of radio antennas for the electromagnetic component and the water-Čerenkov detector for the muons. Thus, $2 \pi$ sky coverage will be reached. Key science questions would be: To isolate protons and conduct proton astronomy to locate the sources of ultra-high-energy cosmic rays, searches for neutrinos and gamma rays at the highest energies, and particle physics at extreme energies.

\section{Acknowledgement}

We are grateful to the organizers of the 2018 ARENA symposium for the great hospitality at the Laboratori Nazionali del Sud of INFN in Catania.

\section{References}

[1] R. Spencer, C. Rapley, Astronomy \& Geophysics 59, 3.2 (2018)

[2] B. Lovell, The story of Jodrell Bank (Oxford University Press, 1968)

[3] J. Jelley, Cherenkov radiation and its applications (Pergamon Press, 1958)

[4] J. Jelley et al., Nature 205, 327 (1967)

[5] N. Porter et al., Nature 213, 110 (1967)

[6] H. Allan, Progress in Elementary Particles and Cosmic Ray Physics (J.G. Wilson and S.G. Wouthuysen eds., North Holland, 1971), p. 169

[7] A. Nelles, et al., Astropart.Phys. 60, 13 (2015)

[8] C. Glaser, S. de Jong, M. Erdmann, J.R. Hörandel, Astropart. Phys. 104, 64 (2019)

[9] T. Huege, Braz.J.Phys. 44, 520 (2014)

[10] O. Ravel et al., Nucl. Instrum. Meth. A518, 213 (2004)

[11] H. Falcke et al., Nature 435, 313 (2005)

[12] T. Huege, Phys. Rept. 620, 1 (2016)

[13] F.G. Schröder, Prog. Part. Nucl. Phys. 93, 1 (2017)

[14] P. Schellart, et al., Astron.Astrophys. 560, A98 (2013)

[15] S. Buitink et al., Nature 531, 70 (2016)

[16] J. Schulz, Pierre Auger Collaboration, Proceedings 34th International Cosmic Ray Conference, Den Haag p. PoS(ICRC2015)615 (2015)

[17] I. Petrov, S. Knurenko, PoS ICRC2017, 335 (2018)

[18] F.G. Schröder et al., JPS Conf. Proc. 9, 010008 (2016)

[19] S.W. Barwick et al., Astropart. Phys. 90, 50 (2017)

[20] A. Nelles et al., JINST 10, P11005 (2015)

[21] A. Aab et al. (Pierre Auger), JINST 12, T10005 (2017)

[22] A. Corstanje et al., Astron. Astrophys. 590, A41 (2016)

[23] A. Aab et al. (Pierre Auger), JINST 11, P01018 (2016)

[24] D. Ardouin et al., Astropart. Phys. 31, 192 (2009)

[25] M. van Haarlem, et al., Astronomy \& Astrophysics 556, A2 (2013)

[26] F. Kahn, I. Lerche, Royal Society of London Proceedings Series A 289, 206 (1966)

[27] G. Askaryan, Journal of the Physical Society of Japan Supplement 17, C257 (1962)

[28] P. Schellart, et al., JCAP 1410, 014 (2014)

[29] A. Aab et al. (Pierre Auger Collaboration), Phys.Rev. D89, 052002 (2014) 
[30] V. Marin (CODALEMA), Charge excess signature in the CODALEMA data. Interpretation with SELFAS2., in Proceedings, 32nd International Cosmic Ray Conference (ICRC 2011) (2011), Vol. 1, pp. 291-294

[31] W.D. Apel et al. (LOPES), Astropart. Phys. 32, 294 (2010)

[32] A. Nelles, et al., JCAP 1505, 018 (2015)

[33] D. Heck et al., Corsika: A monte carlo code to simulate extensive air showers, Report FZKA 6019, Forschungszentrum Karlsruhe (1998)

[34] T. Huege, M. Ludwig, C.W. James, ARENA 2012, AIP Conf. Proc. 1535 pp. 128-132 (2013)

[35] J. Alvarez-Muniz, W.R. Carvalho, Jr., E. Zas, Astropart. Phys. 35, 325 (2012)

[36] A. Corstanje, et al., Astropart.Phys. 61, 22 (2015)

[37] W. Apel, et al., JCAP 1409, 025 (2014)

[38] S. Thoudam, et al., Nucl.Instrum.Meth. A767, 339 (2014)

[39] S. Thoudam et al., Astropart. Phys. 73, 34 (2016)

[40] S. Buitink, et al., Phys.Rev. D90, 082003 (2014)

[41] A. Aab et al. (Pierre Auger), Phys. Rev. D93, 122005 (2016)

[42] A. Aab et al. (Pierre Auger), Phys. Rev. Lett. 116, 241101 (2016)

[43] S. Buitink et al., Proceedings 34th International Cosmic Ray Conference, Den Haag p. PoS(ICRC2015)368 (2015)

[44] F. Canfora et al., Proceedings ARENA 2018 (2018)

[45] P.A. Bezyazeekov et al. (Tunka-Rex), JCAP 1601, 052 (2016)

[46] J. Hörandel, Proceedings ARENA 2018 (2018)

[47] T. Huege et al., EPJ Web Conf. 135, 02003 (2017)

[48] A. Zilles, S. Buitink, T. Huege, EPJ Web Conf. 135, 02004 (2017)

[49] F. Schröder, Proceedings ARENA 2018 (2018)

[50] A. Balagopal V., A. Haungs, T. Huege, F.G. Schroeder, Eur. Phys. J. C78, 111 (2018), [erratum: Eur. Phys. J.C78,no.12,1017(2018)]

[51] J. Alvarez-Muñiz et al. (GRAND) arXiv:1810.09994 (2018)

[52] J. Hörandel, Proceedings UHECR symposium, Paris (2018)

[53] A. Aab et al. (Pierre Auger), arXiv:1604.03637 (2016)

[54] A. Letessier-Selvon, P. Billoir, M. Blanco, I.C. Mariş, M. Settimo, Nucl. Instrum. Meth. A767, 41 (2014) 